\title{
Quasiparticle thermal conductivity in the vortex state of high- $T_{c}$ cuprates.
}

\author{
I. Vekhter ${ }^{a}$ and A. Houghton ${ }^{b}$ \\ ${ }^{a}$ Department of Physics, University of Guelph, Guelph, Ontario N1G 2W1, Canada \\ ${ }^{b}$ Department of Physics, Brown University, Providence, RI 02912-1843, USA
}

(January 9, 2018)

\begin{abstract}
We present the results of a microscopic calculation of the longitudinal thermal conductivity, $\kappa_{x x}$, of a $d$-wave superconductor in the mixed state. Our results show an increase in the thermal conductivity with the applied field at low temperatures, and a decrease followed by a nearly field independent $\kappa_{x x}(H)$ at higher temperatures, in qualitative agreement with the experimental results. We discuss the relationship between the slope of the superconducting gap and the plateau in $\kappa_{x x}(H)$.
\end{abstract}

While the origin of the unusual normal state properties of the high- $\mathrm{T}_{c}$ cuprates remains controversial, many experimental results lend support to the belief that the superconducting state of these materials is relatively mundane, and consists of a pair condensate with an "unconventional" order parameter, believed to be of $d$-wave symmetry, and well-defined quasiparticle excitations above it. However, transport measurements in the vortex state yield some surprising results which question whether the low-temperature physics of the cuprates is well understood.

Perhaps the most notable such example is the observation of a plateau-like feature in the magnetic field dependence of the longitudinal thermal conductivity, $\kappa_{x x}(H)$, in superconducting $\mathrm{Bi}_{2} \mathrm{Sr}_{2} \mathrm{CaCu}_{2} \mathrm{O}_{8+\delta}$ (BSCCO) [1] and $\mathrm{YBa}_{2} \mathrm{Cu}_{3} \mathrm{O}_{7-\delta}$ (YBCO) [2]. At temperatures above a few Kelvin $\kappa_{x x}(H)$ initially decreases with applied magnetic field, $H$, before becoming nearly field independent. [1 -4] The kink and hysteresis in $\kappa_{x x}(H)$ observed in zero-field cooled BSCCO [1,3] have not been found in YBCO [2] or in the field-cooled BSCCO samples [4], and we take the point of view that they are not a generic feature of the $\kappa_{x x}(H)$ dependence in cuprates. In some samples the "plateau" is not reached, and $\kappa_{x x}(H)$ decreases continuously for $H \leq 14$ Tesla, [2, 4, as it does for conventional superconductors for $H \ll H_{c 2}$. [5] This is in sharp contrast to the behavior of the thermal conductivity of the cuprates at ultra-low temperatures, which increases with $H$. [3, 6] Moreover, both features have been observed in the same sample [3], suggesting that the two regimes should be understood within the same approach, which presents a challenge to theories of quasiparticle transport in the vortex state of the cuprates.

Existing theories are based on the observation that, for a single vortex, the properties of superconductors with lines of nodes in the energy gap are dominated by extended quasiparticle states, rather than, as in the $s$-wave case, by localized states in the vortex cores. [7] The magnetic field is taken into account semiclassically, by introducing a Doppler energy shift due to the circulating supercurrents. [7] In one approach physical quantities are computed locally and are averaged over a unit cell of the vortex lattice [8]. The results obtained within this framework describe experimental results quite well 8 10, and the predictions for $\kappa_{x x}(H)$ by Kübert and Hirschfeld [9] fit the ultralow- $T$ measurements. [6] The results of Ref. [9] show a decrease in $\kappa_{x x}(H)$ at higher $T \ll T_{c}$, but not a plateau. A similar approach was used for heavy fermion superconductors. 111

Franz [12] has pointed out that the averaging procedure used in Refs. [8 10] neglected scattering from the vortex lattice. He included the scattering semiphenomenologically, assuming a completely disordered vortex lattice and a Matthiesen type rule for the impurity and vortex contributions to the lifetime 13. He predicted that at any $T \leq T_{c} \kappa_{x x}(H)$ will approach asymptotically the universal value 14, 15] $\kappa_{00}=\pi T N_{0} v^{2} / 6 \Delta_{0}$, where $N_{0}$ is the normal state density of states at the Fermi surface, $v$ is the Fermi velocity, and $\Delta_{0}$ is the gap amplitude. His results are in qualitative agreement with the results of Ref. [2], but the theory requires additional assumptions to explain the low- $T$ limit. Consequently, no existing theory explains both the low- $T$ results and the plateau.

In this work we present a microscopic theory of the thermal conductivity of a two-dimensional clean $\left(l \gg \xi_{0}\right.$, where $l$ is the mean free path, and $\xi_{0}$ is the coherence length) $d$-wave superconductor in the vortex state, with the field perpendicular to the basal plane, and thermal current in the plane. Our results fit the low- $T$ data of Ref. [6] and show a plateau-like feature at higher $T$. We employ the quasiclassical method 16.17, in which the basic quantity is the matrix Green's function integrated over the quasiparticle energy,

$\widehat{g}\left(\phi, \mathbf{R} ; \omega_{n}, \omega_{n^{\prime}}\right)=\int \frac{d \zeta_{p}}{\pi} \widehat{G}\left(p, \mathbf{R} ; \omega_{n}, \omega_{n^{\prime}}\right)=\left(\begin{array}{cc}g & -f \\ f^{\dagger} & \bar{g}\end{array}\right)$.

Here the diagonal elements, $g$ and $\bar{g}$, are the particle and hole propagators, respectively, the off-diagonal elements, Gorkov's anomalous functions $f$ and $f^{\dagger}$, are probability amplitudes for the creation or destruction of a Cooper pair, $\mathbf{R}$ is the center of mass coordinate, $\zeta_{p}$ is the energy of a quasiparticle with momentum $p$ measured relative to the Fermi surface, and $\phi$ is the angle 
parameterizing the cylindrical Fermi surface. The spatial dependence of the vortex lattice (VL) is modeled by an Abrikosov-like solution $\Delta(\mathbf{R}, \phi)=\Delta(\mathbf{R}) \varphi(\phi)$, where $\Delta(\mathbf{R})=\sum_{k_{y}} C_{k_{y}} e^{i k_{y} y} \exp \left(-\left(x-\Lambda^{2} k_{y}\right)^{2} / 2 \Lambda^{2}\right)$, the magnetic length $\Lambda=(2 e H)^{-1 / 2}(\hbar=c=1$ throughout the paper), and $\varphi(\phi)=\cos 2 \phi$ is the angular dependence of the gap. [18

We use the quasiclassical method in the framework proposed in Ref. 20] for $s$-wave superconductors near $H_{c 2}$. In that approach the diagonal elements of $\widehat{g}$ are approximated by their spatial averages, while the exact spatial dependence of the off-diagonal elements is kept. The authors of Ref. [20] noticed that $g$ and $\bar{g}$ are periodic in $\mathbf{R}$ with the periodicity of the vortex lattice, and that Fourier components $g_{\mathbf{K}}$ with reciprocal vortex lattice vectors $\mathbf{K} \neq 0$ are smaller than $g_{\mathbf{K}=0}$ (which is the spatial average of $g$ ) by a factor $\exp \left(-\Lambda^{2} K^{2}\right)$. This approach provides a very accurate description of the vortex state in conventional superconductors. 202 24 The analysis of Ref. [20] relies on the fact that the spatial dependence of the order parameter is given by an Abrikosov-like solution of the linearized Ginzburg-Landau equations, and therefore works especially well in extreme type-II superconductors where it can be extended over almost the entire region of linear magnetization. [25] For an $s$-wave superconductor it breaks down at fields where properties are determined by the states bound to vortex cores. In unconventional superconductors where properties are dominated by extended states [7] the approach works down to fields close to $H_{c 1}$, i.e. for all experimentally relevant fields. 19.

The leading order quasiclassical propagator is determined from equations [23, 26]

$$
\begin{aligned}
\left(2 \widetilde{\omega}_{n}+\mathbf{v}(\nabla-2 i e \mathcal{A})\right) f & =2 i \Delta(\mathbf{R}) g \cos 2 \phi \\
\left(2 \widetilde{\omega}_{n}-\mathbf{v}(\nabla+2 i e \mathcal{A})\right) f^{\dagger} & =2 i \Delta^{\star}(\mathbf{R}) g \cos 2 \phi,
\end{aligned}
$$

complemented by the normalization condition $g^{2}-f f^{\dagger}=$ -1 . Here $\mathcal{A}=(0, H x, 0)$, and the renormalized frequency, $i \widetilde{\omega}_{n}=i \omega_{n}+i \sigma\left(i \widetilde{\omega}_{n}\right)$, depends on the self-energy due to impurity scattering, $\sigma$, which is determined within the $t$-matrix approximation [27]. We ignore corrections to the impurity vertex due to the magnetic field, which is justified when $\Lambda \ll l$. Since $\Lambda \simeq 183 \AA / \sqrt{H \text {, Tesla, for }}$ clean samples this condition is satisfied at least above 0.1-0.5 T.

The solution of Eqs.(11),(2) is known to be [23,26 $g=-i \operatorname{sgn}\left(\omega_{n}\right) P\left(\phi, \widetilde{\omega}_{n}\right)$, where $P\left(\phi, \widetilde{\omega}_{n}\right)=[1-$ $\left.i \sqrt{\pi}(2 \Lambda \Delta / v)^{2} W^{\prime}\left(u_{n}\right) \cos ^{2} 2 \phi\right]^{-1 / 2}$, the function $W(u)=$ $e^{-u^{2}} \operatorname{erfc}(-i u), u_{n}=2 i \widetilde{\omega}_{n} \Lambda \operatorname{sgn}\left(\omega_{n}\right) / v$, and $\Delta$ is the spatial average of the amplitude of the order parameter. In the limit $H=0$ setting $\Delta$ equal to the gap amplitude, $\Delta_{0}$, we recover the BCS Green's function and consequently all the results of the standard "dirty $d$-wave" theory. 28] For a pure sample at low fields the residual density of states (DOS) $N(0) / N_{0}=\left(v / \Lambda \Delta_{0} \pi \sqrt{2}\right) \ln \left(8 \sqrt{2} \Lambda \Delta_{0} / v\right) \propto$
$\sqrt{H} \ln \left(H_{c 2} / H\right)$; the field dependence differs by a logarithm from the single vortex result of Volovik [7]. For the range of fields where comparison with experiment has been made the logarithm is nearly constant, and the result for the DOS agrees with that of of Ref. [8] up to a numerical factor. In the following we consider scattering in the unitarity and Born limits, so that the parameters of the theory are the normal state scattering rate, $\Gamma$, and the dimensionless quantity $\Lambda \Delta / v$. The latter can be estimated by setting $\Delta \simeq \Delta_{0}\left(1-H / H_{c 2}\right)^{1 / 2}$, and noticing that $\Lambda \Delta_{0} / v=\left(\Lambda k_{f} / 2\right)\left(v_{2} / v\right)$, where $v_{2}$ is the slope of the gap at the node. In BSCCO $k_{f} \simeq 0.737$ $\AA^{-1}$, and is relatively doping independent, [29] then $\Lambda \Delta / v \sim \alpha \sqrt{\left(1-H / H_{c 2}\right) / H}$; where, for field in Tesla, $\alpha \sim 3.5$ for the overdoped case, where $v / v_{2} \sim 20$, but becomes smaller on the underdoped side. 30 In YBCO, taking $k_{f} \sim 0.8 \AA^{-1}$ 31]) we obtain $\alpha \sim 5$ if $v / v_{2} \sim 14$ [6], or twice that value if $v / v_{2} \sim 7$. [32]

The heat current can now be determined from the linear response equations 24.26]

$$
\begin{aligned}
\mathbf{j}_{h}= & \pi N_{0} \lim _{i \omega_{0} \rightarrow i 0^{+}} \sum_{\omega_{n}} \int d \phi \mathbf{v}(\phi)(\mathbf{v}(\phi) \cdot \nabla T) \\
& \times \omega_{n}\left(\omega_{n}-\omega_{0}\right) \frac{g\left(\omega_{n}\right)-g\left(\omega_{n}-\omega_{0}\right)}{i \widetilde{\omega}_{0}+2 b}
\end{aligned}
$$

where $b=\left(\Delta f^{\dagger}\left(\omega_{n}\right)+\Delta^{\star} f\left(\omega_{n}-\omega_{0}\right)\right) /\left(g\left(\omega_{n}\right)-g\left(\omega_{n}-\right.\right.$ $\left.\left.\omega_{0}\right)\right)$. This leads to the thermal conductivity

$$
\begin{aligned}
\frac{\kappa_{x x}}{T}= & \frac{N_{0} v^{2}}{4 \pi} \int_{0}^{\infty} \frac{d \omega}{T}\left(\frac{\omega}{T}\right)^{2} \cosh ^{-2}\left(\frac{\omega}{2 T}\right) \\
& \times \int_{0}^{2 \pi} d \phi \cos ^{2} \phi[\operatorname{Re} P(\widetilde{\omega})] \tau(\widetilde{\omega}, \phi), \\
\frac{1}{2 \tau(\widetilde{\omega}, \phi)} & =\operatorname{Re} \sigma(\widetilde{\omega}) \\
& +2 \sqrt{\pi} \frac{\Lambda \Delta^{2}}{v} \frac{\operatorname{Re}[P(\widetilde{\omega}) W(u)]}{\operatorname{Re} P(\widetilde{\omega})} \cos ^{2} 2 \phi .
\end{aligned}
$$

Since $\operatorname{Re}[P(\widetilde{\omega}, \phi)]$ is the angular dependent DOS, and $\tau(\widetilde{\omega}, \phi)$ is a transport lifetime, Eqs.(何) and (5) generalize the normal state expression $\kappa_{x x} \equiv \kappa_{n}=\pi^{2} N_{0} v^{2} T \tau / 6$. There is a contribution to the quasiparticle lifetime from Andreev scattering off the vortex lattice, which has the same symmetry on the Fermi surface as the gap function, and is therefore the more important the higher the quasiparticle energy. This contribution appears even for a periodic vortex lattice, since Bloch's theorem only forbids scattering from a periodic potential in the absence of impurity broadening of the quasiparticle wave-packet, not in the presence of impurities. Note that the dependence of the DOS and the scattering rate on angle cannot be disentangled, in contrast to the assumption of Ref. [12]. Also, it is clear from the form of Eq.(化) that a simple scaling form as a function of $T / \sqrt{H}$ cannot be expected.

First, we consider the limit $T=0$, where analytic results can be obtained. It is convenient to analyze the 
thermal conductivity in terms of a transport lifetime $\tau_{H}$, defining $\kappa_{x x}(H) / T=\pi^{2} N_{0} v^{2} \tau_{H} / 6$, and expressing $\tau_{H}$ in terms of the lifetime $\tau_{\text {eff }} \equiv 1 /(2 \gamma)$, where $\gamma=\sigma(\omega=0)$ is the zero-frequency scattering rate. For $H=0$ we obtain $\tau_{H}=k E(k) / \pi \Delta_{0}$, where $E$ is the complete elliptic integral, and $k=\left(1+\left(2 \Delta_{0} \tau_{\text {eff }}\right)^{-2}\right)^{-1 / 2}$ in agreement with Refs. 33, 15]. For $\Delta_{0} \tau_{\text {eff }} \gg 1$ we recover the universal limit $\kappa_{x x}=\kappa_{00} \equiv \pi T N_{0} v^{2} / 6 \Delta_{0}$. [14, 15] At finite fields the integral in Eq.(14) is evaluated approximately to give $\tau_{H} \simeq\left(\tau_{\text {eff }} v / 4 \sqrt{\pi} \Lambda \Delta_{0}^{2}\right)^{1 / 2}$. In the "clean" limit $v / \Lambda \gg \gamma$ for the important cases of Born $\left(\Gamma_{B}\right)$ and unitarity $\left(\Gamma_{U}\right)$ scattering, we find

$$
\tau_{H} \simeq\left\{\begin{array}{l}
\left(\frac{\pi}{32}\right)^{1 / 4}\left(\Gamma_{B} \Delta_{0}\right)^{-1 / 2} \ln ^{-1 / 2}\left(\frac{8 \sqrt{2} \Lambda \Delta_{0}}{v}\right) \\
\left(128 \pi^{3}\right)^{-1 / 4}\left(\Gamma_{U} \Delta_{0}\right)^{-1 / 2}\left(\frac{v}{\Lambda \Delta_{0}}\right) \ln ^{1 / 2}\left(\frac{8 \sqrt{2} \Lambda \Delta_{0}}{v}\right) .
\end{array}\right.
$$

This expression is in a good agreement with the numerical results at intermediate fields. In Fig. 1 we use the numerical evaluation of Eq. (1) to compare $\kappa_{x x}(H)$ for Born and unitarity scattering. In the case of Born scattering $\kappa_{x x}(H)$ only reaches the universal limit at exponentially small fields, and is approximately field-independent at higher $H$, Eq.(6). In the unitarity limit, on the other hand, $\kappa_{x x}(H)$ increases approximately as $\sqrt{H}$ at low fields and the approach to $\kappa_{00}$ is clearly seen. At low $T$ it is seen that $\kappa_{x x}(H)$ tends to $\kappa_{00}$ over a field range of order a few Tesla [6], and that the field dependence is close to $\sqrt{H}$ at low $T$. [3] This suggests that impurity scattering is close to the unitarity limit at low temperatures, and we consider this limit hereafter. The $H$ dependence of $\tau_{H}$ differs from that found in the Doppler shift theory [9], however, since $\sqrt{\ln H}$ varies slowly, for experimentally accessible fields the behavior of $\kappa_{x x}(H)$ is close in to that obtained by Kübert and Hirschfeld. [9] In Fig. 2 we fit the data of Ref. [6]. Using the the value of $v / v_{2}=14$ from Refs. [6. 34] we find $\Gamma$ to be about 4 times greater than the estimate of Ref. [6]. In the present theory $\kappa_{x x}(H)$ increases faster with field the smaller the slope of the gap, approximately as $\sqrt{H / \alpha^{2} \Gamma}$, see Eq. (6), so that the same data can be fit on assuming a smaller impurity concentration if we allow for a larger ratio $v_{2} / v$. [32] This also implies that a smaller slope of the gap can result in a larger increase in $\kappa_{x x}(H)$ for a dirtier sample, see Fig. 2, which may be a part of the explanation of a faster increase of $\kappa_{x x}(H)$ in nominally dirtier BSCCO compared to YBCO. [3]

At finite temperatures the integration in Eq.(14) has to be carried out numerically; we confine ourselves to the regime where the gap is temperature independent. We have checked and find agreement with previous results at $H=0$. 15] $\kappa(0) / T$ increases rapidly for $T \gg \gamma$, and for clean samples at $T \sim 0.1 \Delta_{0}$ it exceeds $\kappa_{00}$ by two orders of magnitude. At the same time at strong fields, $v / \Lambda \gg T, \kappa_{x x}(H) / T$ is controlled by the field strength and is only weakly temperature dependent. The field dependence at $T=0$ is weak, close to $\sqrt{H}$, so that for $H \sim 10 \mathrm{~T} \kappa_{x x}(H) / T \leq 10 \kappa_{00} / T$. An immediate conclusion is that for $T>\gamma$ there is a steep drop in $\kappa_{x x}(H)$ at low fields, followed by a crossover to the $\sqrt{H}$ like behavior. This crossover occurs when $v / \Lambda \sim T$, at a field $H^{*} \propto T^{2}$, as indicated experimentally. [1, 3]

In Fig.3 we show the results: at high $T$ the ratio $\kappa_{x x}(H) / \kappa_{x x}(0)$ decreases continuously as a function of field, however as the temperature is lowered a plateau develops, and at even lower $T$ a pronounced minimum appears in the field dependence. As the lower panel demonstrates there is a transition to an increasing $\kappa_{x x}(H)$ at the lowest temperatures. Numerically, we find that at fixed $T$ a smaller value of $\alpha$ corresponds to a more pronounced plateau in $\kappa_{x x}(H)$. This implies that the nearly field independent thermal conductivity should be more evident in the underdoped cuprates. [30]

To summarize, we give a theory of the thermal conductivity of a $d$-wave superconductor in the vortex state as a function of field at $T \ll T_{c}$. In zero field and at low $T$ we recover the universal results [14,15. We find that there exists a contribution to the transport lifetime due to scattering off the vortices, which has the same symmetry as the gap function. Consequently, at low temperatures when most of the quasiparticles are near the gap nodes it plays a minor role and the effect of the field is to increase the DOS and the thermal conductivity. In contrast, at high $T$ the main effect of the vortices is to introduce a new scattering mechanism, and $\kappa_{x x}(H)$ decreases sharply to a plateau-like feature at higher fields (a broad minimum); the crossover field scales with $T^{2}$. This behavior is in agreement with experimental results. [1 4,6$]$. Finally, it is important to compare the predictions of our theory with the measurements of the transverse (Hall) thermal conductivity, $\kappa_{x y}$, [2] to which phonons do not contribute. At low fields $\kappa_{x y}$ is given by Eq. (4) with the kernel $\tau_{x y}(\widetilde{\omega}, \phi)=\omega_{c} \tau(\widetilde{\omega}, \phi) / 2 \operatorname{Re}[\sigma(\widetilde{\omega})]$. However, as the measurements are taken at $T \geq 20 \mathrm{~K}$, in a regime where it is necessary to include inelastic scattering in the analysis of both longitudinal and transverse thermal conductivity, [35] we defer a discussion of this issue until later.

We are grateful to M. Chiao, M. Franz, D. Maslov, and especially P. J. Hirschfeld and L. Taillefer for discussions, and to K. Behnia for correspondence. A.H. acknowledges the hospitality of ITP Santa Barbara, where part of this work was done.

[1] K. Krishana et al. Science 277, 83 (1997).

[2] N. P. Ong et al., cond-mat/9904160

[3] H. Aubin et al., Phys. Rev. Lett. 82, 624 (1999). 
[4] Y. Ando et al., cond-mat/9812265.

[5] J. Lowell and J. B. Sousa, J. Low Temp. Phys. 3, 65 (1970).

[6] M. Chiao et al., Phys. Rev. Lett. 82, 2943 (1999).

[7] G. E. Volovik, JETP Lett. 58, 469 (1993).

[8] C. Kübert and P. J. Hirschfeld, Sol. St. Comm. 105. 459 (1998).

[9] C. Kübert and P. J. Hirschfeld, Phys. Rev. Lett. 80, 4963 (1998).

[10] I. Vekhter, J. P. Carbotte, and E. J. Nicol, Phys. Rev. B 59, 1417 (1999).

[11] Yu. S. Barash, V. P. Mineev, and A. A. Svidzinskii, JETP Lett. 65, 638 (1997); Yu. S. Barash and A. A. Svidzinskii, Phys. Rev. B 58, 6476 (1998).

[12] M. Franz, Phys. Rev. Lett. 82, 1760 (1999).

[13] F. Yu et al., Phys. Rev. Lett. 74, 5136 (1995).

[14] P. A. Lee, Phys. Rev. Lett. 71, 1887 (1993)

[15] M. J. Graf et al., Phys. Rev. B 53, 15147 (1996).

[16] G. Eilenberger, Z. Phys. 214, 195 (1968).

[17] A. I. Larkin and Yu. N. Ovchinnikov, Zh. Exp. Teor. Fiz. 55, 2262 (1968) [Sov. Phys. JETP 28, 1200 (1969)].

[18] We ignore here the small (less than 3\% [19]) contribution to the spatial dependence of $\Delta(\mathbf{R})$ of the higher Landau level components, analysed in I. A. Luk'yanchuk and V. P. Mineev, Zh. Eskp. Teor. Fiz 93, 2030 (1987) [Sov. Phys. - JETP 66, 1168 (1987)].

[19] H. Won and K. Maki, Phys. Rev. B 53, 5927 (1996).

[20] U. Brandt, W. Pesch, and L. Tewordt, Z. Phys. 201, 209 (1967).

[21] A. Houghton and K. Maki, Phys. Rev. B 4, 843 (1971); ibid. 4, 847 (1971).

[22] M. K. Purvis , R. A. Johnson, and A. R. Hoffmann, J. Phys. Chem Solids 35, 989 (1974).

[23] W. Pesch, Z. Phys. B 21, 263 (1975).

[24] P. Klimesch and W. Pesch, J. Low Temp. Phys. 32, 869 (1978).

[25] E. H. Brandt, Rep. Prog. Phys. 58, 1465 (1995).

[26] A. Houghton and I. Vekhter, Phys. Rev. B 57, 10831 (1998).

[27] P. J. Hirschfeld, D. Vollhardt, and P. Wölfle, Solid State Commun. 59, 111 (1986); S. Schmitt-Rink, K. Miyake, and C. M. Varma, Phys. Rev. Lett. 57, 2575 (1986).

[28] P. J. Hirschfeld and N. Goldenfeld, Phys. Rev. B 48, 4219 (1993).

[29] H. Ding et al. Phys. Rev. Lett. 78, 2628 (1997).

[30] J. Mesot et al., cond-mat/9812377

[31] M. C. Schabel et al., Phys. Rev. B 57, 6090 (1998).

[32] P. A. Lee and X.-G. Wen, Phys. Rev. Lett. 78, 4111 (1997).

[33] Y. Sun and K. Maki, Europhys. Lett. 32, 355 (1995).

[34] L. Taillefer et al. Phys. Rev. Lett. 79, 483 (1997).

[35] P. J. Hirschfeld, private communication; P. J. Hirschfeld and W. O. Putikka, Phys. Rev. Lett. 77, 3909 (1996).

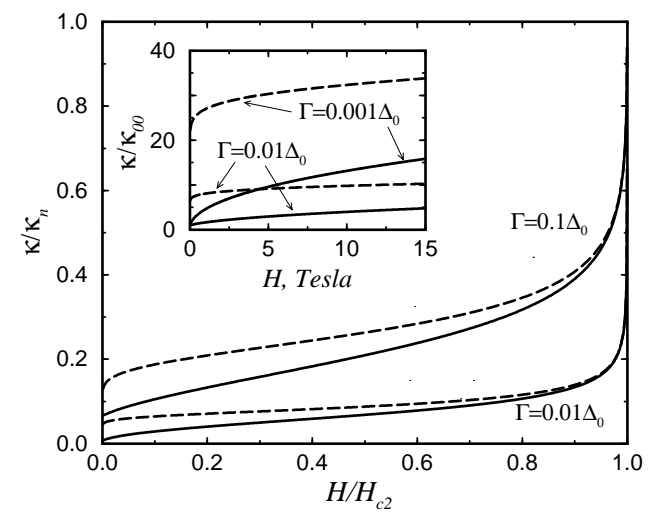

FIG. 1. Field dependence of the thermal conductivity at $T=0$ for scattering in the unitarity (solid lines) and Born (dashed lines) limits. Inset: low field behavior, normalized by the universal value $\kappa_{00} . H_{c 2}=150 \mathrm{~T}$.

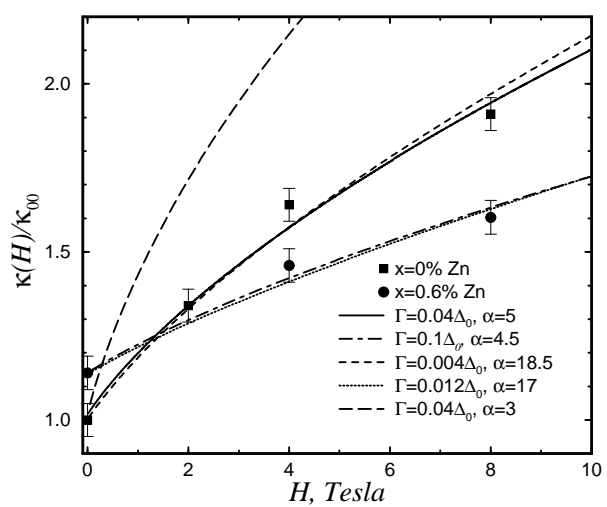

FIG. 2. Field dependence of $\kappa_{x x}(H)$ at $T=0$ compared to data from Ref.[6].

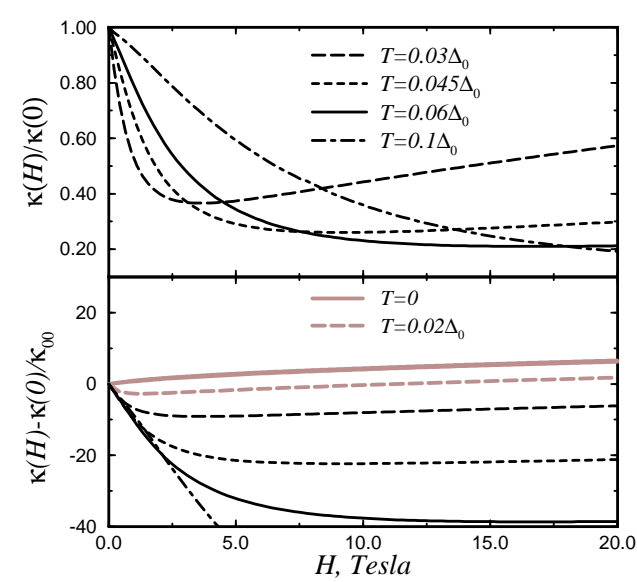

FIG. 3. Top: field dependence of $\kappa_{x x}(H) / \kappa(0)$ for $\Gamma=0.003 \Delta_{0}$ and $\alpha=7$ at temperatures where $\kappa_{x x}(H)$ is decreasing; bottom: the change in the thermal conductivity as a function of field for different temperatures. 